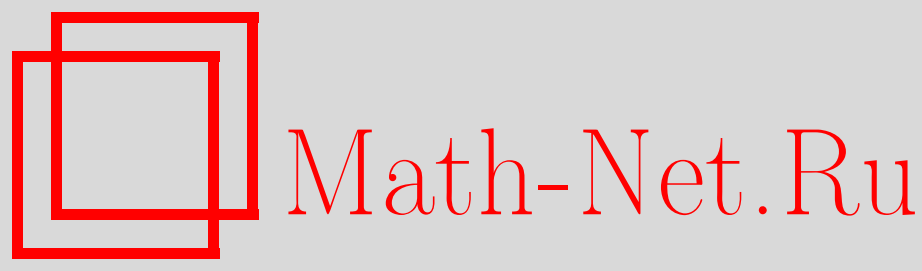

Е. А. Яревский, Асимптотическое поведение волновой функции системы нескольких частиц с растущими на бесконечности парными взаимодействиями, ТMФ, 2016, том 186, номер 1, 164-176

DOI: https://doi.org/10.4213/tmf8976

Использование Общероссийского математического портала Math-Net.Ru подразумевает, что вы прочитали и согласны с пользовательским соглашением http://www . mathnet.ru/rus/agreement

Параметры загрузки :

IP : 35.174 .16 .151

26 апреля 2023 г., 17:00:18

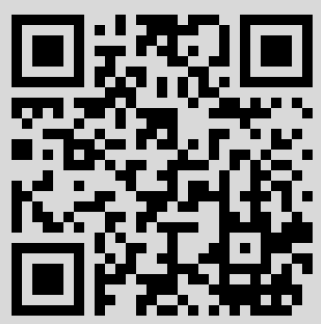




\title{
АСИМПТОТИЧЕСКОЕ ПОВЕДЕНИЕ ВОЛНОВОЙ ФУНКЦИИ СИСТЕМЫ НЕСКОЛЬКИХ ЧАСТИЦ С РАСТУЩИМИ НА БЕСКОНЕЧНОСТИ ПАРНЫМИ ВЗАИМОДЕЙСТВИЯМИ
}

\begin{abstract}
Построена асимптотика волновых функций систем двух и трех квантовых частиц с растущими на бесконечности парными взаимодействиями. Рассмотрены трехчастичные системы на прямой и в трехмерном пространстве. Уравнения эйконала и переноса, использованные для построения асимптотик, существенно отличаются от уравнений для случая убывающих потенциалов. Подробно исследовано решение нелинейного уравнения эйконала.
\end{abstract}

Ключевые слова: конфайнмент, уравнение эйконала, асимптотика волновой функции. DOI: $10.4213 / \operatorname{tmf} 8976$

Работа посвящена памяти академика С. П. Меркуръева в год 70-летия со дня его рождения

\section{1. ВВЕДЕНИЕ}

Изучение асимптотик волновых функций является одним из важных методов исследования свойств квантовых систем нескольких частиц [1]. Для трехчастичных систем координатные асимптотики волновых функций подробно исследованы и для функций непрерывного спектра [1], и для связанных состояний [2]. Полученные асимптотики используются как для решения задачи рассеяния, так и для увеличения эффективности поиска собственных значений и собственных функций квантовых систем [3]. Эти асимптотики описывают волновые функции систем с убывающими на больших расстояниях парными взаимодействиями. Однако существуют задачи, в которых потенциалы растут на больших расстояниях. В частности, такие задачи встречаются при изучении моделей элементарных частиц [4]-[6], при исследовании поведения ионов в электромагнитных ловушках [7], движения электронов

Работа была частично поддержана РФФИ (грант № 14-02-00326) и Санкт-Петербургским государственным университетом (проект № 11.38.241.2015).

${ }^{*}$ Санкт-Петербургский государственный университет, Санкт-Петербург, Россия. E-mail: e.yarevsky@spbu.ru 
в гетероструктурах [8] и при решении уравнения Солпитера с потенциалом, зависящим от времени [9]. Асимптотическое поведение волновых функций для растущих потенциалов известно значительно хуже [10], [11].

Асимптотики волновых функций могут быть исследованы с помощью метода эйконала [1], [12]. Однако для потенциалов, убывающих или растущих на бесконечности, имеется существенное различие в свойствах нелинейного уравнения эйконала. В случае убывающих потенциалов кинетическая энергия преобладает над потенциальной на больших расстояниях, и уравнение можно асимптотически проинтегрировать. Для систем с растущими потенциалами потенциальной энергией пренебречь нельзя, и ее сложное поведение на больших расстояниях разрушает симметрию уравнения эйконала. Таким образом, поведение решения уравнения эйконала (и, соответственно, волновой функции) становится более сложным. Как будет показано ниже, известный пример гармонического осциллятора не отражает всю возможную сложность волновой функции, так как само по себе квадратичное взаимодействие обладает высокой симметрией, и решение в этом случае можно найти в явной форме.

Асимптотика волновой функции для системы трех частиц с растущими взаимодействиями на прямой была получена в работе [11]. Однако существование решения нелинейного уравнения эйконала было доказано только для некоторых специальных значений степени конфайнмента. Кроме того, в приложениях, как правило, требуется асимптотика волновой функции не в одномерном, а в обычном трехмерном пространстве [4], [13]. В настоящей работе сначала на более простом одномерном примере трех частиц предложен подход, позволяющий доказать существование решения при произвольной степени конфайнмента. Затем этот подход применяется к системе трех частиц в трехмерном пространстве. Нужно отметить также, что энергетический спектр системы с растущими парными взаимодействиями весьма прост: он является чисто дискретным, и каждое значение имеет конечную кратность. Асимптотику спектра можно вычислить с помощью квазиклассического метода, однако такое вычисление находится вне рамок настоящей работы.

Работа организована следующим образом. В разделе 2 построена асимптотика волновой функции для системы двух частиц в трехмерном пространстве. Это простое вычисление позволяет ввести необходимые обозначения и иллюстрирует поведение волновой функции в более сложных случаях. В разделе 3 обсуждается общая схема построения асимптотики волновой функции для системы трех частиц. В разделе 4 доказано существование решения уравнения эйконала для системы трех частиц в $\mathbb{R}^{1}$. В разделе 5 построено асимптотическое разложение волновой функции для системы трех частиц в трехмерном пространстве. В разделе 6 обсуждаются некоторые особенности полученных решений.

\section{2. АСИМПТОТИКА ВОЛНОВОЙ ФУНКЦИИ ДЛЯ СИСТЕМЫ ДВУХ ЧАСТИЦ}

В данном разделе для двухчастичной квантовой системы вычислена асимптотика волновой функции на больших расстояниях для потенциалов, ведущих себя асимптотически степенным образом. В данном случае асимптотики могут быть получены с использованием стандартного квазиклассического подхода. 
Рассмотрим двухчастичное уравнение Шредингера в пространстве $\mathbb{R}^{3}$ :

$$
(-\Delta+V(|x|)-E) \psi(x)=0,
$$

где $V$ - сферически-симметричный потенциал, имеющий вид

$$
V(|x|)=\lambda|x|^{n}+W(|x|), \quad \lambda>0, \quad n>0 .
$$

Предполагается, что потенциал $W(|x|)$ убывает на больших расстояниях так, что функция $|x| W(|x|)$ ограничена при $|x| \rightarrow \infty$, и $W(|x|)$ является достаточно гладким. Таким образом, $V(|x|)$ растет на больших расстояниях, как степенная функция. Такого предположения о поведении потенциала достаточно для приложений. Возможность обобщения полученных результатов на случай другого поведения потенциала $W(|x|)$ кратко обсуждается в заключении.

В сферических координатах $x=\{\rho=|x|, \hat{x}\}$ переменные в уравнении (1) разделяются, так что его решение можно искать в виде $\psi_{l m}(x)=\rho^{-1} \varphi_{l m}(\rho) Y_{l}^{m}(\hat{x})$, где $Y_{l}^{m}(\hat{x})$ - сферические гармоники [14], а $l$ и $m$ - угловой момент системы и его проекция соответственно. Функция $\varphi_{l m}(\rho)$ удовлетворяет уравнению

$$
\left(-\frac{d^{2}}{d \rho^{2}}+\frac{l(l+1)}{\rho^{2}}+V(\rho)-E_{l m}\right) \varphi_{l m}(\rho)=0 .
$$

Будем искать асимптотику волновой функции $\varphi_{l m}(\rho) \equiv \varphi(\rho)$ при $\rho \rightarrow \infty$ в представлении эйконала [12]:

$$
\varphi(\rho)=A(\rho) e^{-S(\rho)} .
$$

Подставляя это представление в уравнение (3), получаем в старшем порядке по $\rho \rightarrow \infty$ уравнение эйконала

$$
-\left(S^{\prime}(\rho)\right)^{2}+V_{l}(\rho)-E=0,
$$

где $V_{l}(\rho)=V(\rho)+l(l+1) / \rho^{2}$. Следующий порядок разложения по $\rho$ дает уравнение непрерывности

$$
A(\rho) S^{\prime \prime}(\rho)+2 S^{\prime}(\rho) A^{\prime}(\rho)=0 .
$$

Решение уравнения эйконала (5) можно найти точно,

$$
S(\rho)=\int_{\rho_{0}}^{\rho} \sqrt{V_{l}(\rho)-E} d \rho .
$$

Его асимптотическое разложение при $\rho \rightarrow \infty$ выглядит как

$$
S(\rho)=\sqrt{\lambda} \sum_{m=0}^{M}\left(\begin{array}{c}
1 / 2 \\
m
\end{array}\right)\left(-\frac{E}{\lambda}\right)^{m} \frac{\rho^{1+n / 2-m n}}{1+n / 2-m n}+O(1),
$$


где $\left(\begin{array}{c}1 / 2 \\ m\end{array}\right)$ - биномиальные коэффициенты [14] и

$$
M=\left[\frac{1}{2}+\frac{1}{n}\right]
$$

В последнем соотношении символом $[p]$ обозначена целая часть числа $p$. При принятых предположениях об убывании $W(\rho)$ этот потенциал не влияет на асимптотическое поведение эйконала. Асимптотика эйконала также не зависит явно от углового момента $l$, хотя такая зависимость и может возникать через значения энергии $E$.

Разложение (8) должно быть модифицировано в случае степеней конфайнмента $n=2 /(2 k-1), k \in \mathbb{N}$. В данном случае последнее слагаемое в разложении (8) становится логарифмическим:

$$
S(\rho)=\sqrt{\lambda} \sum_{m=0}^{M-1}\left(\begin{array}{c}
1 / 2 \\
m
\end{array}\right)\left(-\frac{E}{\lambda}\right)^{m} \frac{\rho^{1+n / 2-m n}}{1+n / 2-m n}+\sqrt{\lambda}\left(\begin{array}{c}
1 / 2 \\
M
\end{array}\right)\left(-\frac{E}{\lambda}\right)^{M} \ln \rho+O(1) \text {. }
$$

Решение уравнения непрерывности (6) также можно найти в явном виде:

$$
A(\rho)=\frac{\text { const }}{\sqrt{S^{\prime}(\rho)}}=\frac{\text { const }}{\sqrt[4]{V_{l}(\rho)-E}} .
$$

В старшем порядке по $\rho$ амплитуда $A(\rho)$ записывается как

$$
A(\rho)=\frac{\text { const }}{\sqrt[4]{\lambda}} \rho^{-n / 4}\left(1+O\left(\rho^{-n}\right)\right)
$$

Уравнения (4), (8), (12) описывают асимптотику волновой функции $\varphi(\rho)$ при $\rho \rightarrow \infty$.

\section{3. СХЕМА ПОСТРОЕНИЯ АСИМПТОТИЧЕСКОГО РАЗЛОЖЕНИЯ ВОЛНОВОЙ ФУНКЦИИ ДЛЯ СИСТЕМ ТРЕХ ЧАСТИЦ}

В этом разделе описана общая схема построения асимптотического разложения волновой функции, которая затем применяется к системам трех частиц на прямой и в трехмерном пространстве.

Пусть $X=\left\{x_{\beta}, y_{\beta}\right\}$ - координаты Якоби в паре с номером $\beta, \beta=1,2,3$ [1]. Для трехчастичной системы в одномерном пространстве $\mathbb{R}^{1}$ координаты $x_{\beta}$ и $y_{\beta}$ являются одномерными, а для системы в трехмерном пространстве $\mathbb{R}^{3}$ - трехмерными. Разные системы координат связаны ортогональным преобразованием,

$$
\left(\begin{array}{l}
x_{\gamma} \\
y_{\gamma}
\end{array}\right)=\left(\begin{array}{rr}
c_{\gamma \beta} & s_{\gamma \beta} \\
-s_{\gamma \beta} & c_{\gamma \beta}
\end{array}\right)\left(\begin{array}{l}
x_{\beta} \\
y_{\beta}
\end{array}\right), \quad c_{\gamma \beta}^{2}+s_{\gamma \beta}^{2}=1 .
$$

Коэффициенты $c_{\gamma \beta}, s_{\gamma \beta}$ зависят только от масс частиц [1], [15]. Уравнение Шредингера для волновой функции $\psi(X)$ системы трех частиц с энергией $E$ записывается как

$$
\left(-\Delta_{x_{\beta}}-\Delta_{y_{\beta}}+V(X)-E\right) \psi(X)=0
$$


Парные взаимодействия в трехчастичной системе выбираются такими же, как в двухчастичной системе (2):

$$
v_{\beta}\left(x_{\beta}\right)=\lambda_{\beta}\left|x_{\beta}\right|^{n}+w_{\beta}\left(\left|x_{\beta}\right|\right), \quad \lambda_{\beta}>0, \quad n>0,
$$

причем $\left|x_{\beta}\right| w_{\beta}\left(\left|x_{\beta}\right|\right) \leqslant$ const при $\left|x_{\beta}\right| \rightarrow \infty$. Полный потенциал $V(X)$ является суммой парных потенциалов:

$$
V(X)=\sum_{\beta=1}^{3} v_{\beta}\left(x_{\beta}\right)=\sum_{\beta=1}^{3} \lambda_{\beta}\left|x_{\beta}\right|^{n}+\sum_{\beta=1}^{3} w_{\beta}\left(\left|x_{\beta}\right|\right) .
$$

Будем искать решение уравнения Шредингера (14) в представлении эйконала [12]:

$$
\psi(X)=A(X) e^{-S(X)}
$$

Подставляя это представление в уравнение Шредингера, в старшем порядке по $|X| \rightarrow \infty$ получаем уравнение эйконала

$$
-|\nabla S(X)|^{2}+V(X)-E=0
$$

Следующий порядок по $|X|$ дает уравнение непрерывности

$$
2(\nabla A, \nabla S)+A \Delta S=0
$$

Следует отметить, что это уравнение (как и уравнение эйконала) отличается от уравнений переноса для убывающих потенциалов [1], [12]: в него входит $A \Delta S$, а не $\Delta A$. Дело в том, что эйконал в нашем случае асимптотически растет, а амплитуда асимптотически убывает. Таким образом, основной вклад в уравнение на больших расстояниях вносят именно слагаемые, в которых присутствует эйконал.

Анализ уравнения (19) сравнительно несложен. Действительно, вне окрестности $A(X)=0$ его можно переписать в виде

$$
\nabla \cdot\left(A^{2}(X) \nabla S(X)\right)=0
$$

Ограниченное решение $\vec{u}$ уравнения $\nabla \cdot \vec{u}=0$ является постоянным вектором в старшем порядке по $|X|$ при $|X| \rightarrow \infty$. Следовательно, решение уравнения (20) удовлетворяет в старшем порядке уравнению $A^{2}(X) \nabla S(X)=\vec{u}$. Используя уравнение эйконала (18), получим в старшем порядке

$$
A(X)=\frac{\text { const }}{\sqrt[4]{V(X)-E}} .
$$

Модуль в последнем соотношении опущен, так как волновая функция связанного состояния всегда может быть выбрана вещественной. Нужно отметить, что в случае исследования задачи рассеяния это не так: амплитудная функция $A(X)$ является комплексной и может включать нетривиальный фазовый множитель [1]. Так как 
потенциал $V(X)$ неограниченно возрастает при $|X| \rightarrow \infty$ для любого направления $X$ (см. неравенство (28) ниже), у амплитуды $A(X)$ отсутствуют особые направления.

Решение уравнения эйконала (18) представляет собой существенно более сложную задачу. Даже для случая убывающего потенциала, $|V(X)| \rightarrow 0$ при $|X| \rightarrow \infty$, процесс решения уравнения $|\nabla S|^{2}+E=0$ является достаточно сложным, а само решение имеет существенно разное поведение в различных областях конфигурационного пространства [1]. Когда потенциал $V(X)$ растет на больших расстояниях, уравнение (18) асимптотически принимает вид $|\nabla S|^{2}=V(X)$. Это уравнение не обладает никакими специфическими симметриями, так что его решение не может вести себя проще, чем решение уравнения с убывающими потенциалами. Исследование уравнения (18) является основной задачей настоящей работы.

Перейдем в уравнении Шредингера к гиперсферическим координатам $X=\{\rho, \widehat{X}\}$, где $\rho=|X|$ - гиперрадиус, а $\widehat{X}$ содержит все угловые переменные [15]. Гамильтониан системы трех частиц с нулевым полным угловым моментом имеет в этих координатах вид

$$
H=-\partial_{\rho}^{2}-p \rho^{-1} \partial_{\rho}+\rho^{-2} \square+V(\rho, \widehat{X}),
$$

где потенциал $V(X)$ можно записать как

$$
V(X)=\rho^{n} \alpha(\widehat{X})+\sum_{\beta=1}^{3} w_{\beta}\left(\left|x_{\beta}\right|\right) .
$$

Для системы трех частиц в $\mathbb{R}^{1}$ мы имеем $p=1$, а оператор $\square$ действует как

$$
\square=-\partial_{\varphi}^{2}, \quad \varphi \in[0,2 \pi] .
$$

Функция $\alpha(\widehat{X})$ имеет вид

$$
\alpha(\widehat{X})=\alpha_{1}(\varphi)=\sum_{k=1}^{3} \lambda_{k}\left|\sin \left(\varphi-\varphi_{k}\right)\right|^{n} .
$$

Углы $\varphi_{k}$ определяются коэффициентами $c_{\gamma \beta}, s_{\gamma \beta}[11]$.

Для системы трех частиц в $\mathbb{R}^{3}$ мы имеем $p=5$, а определение оператора $\square$ можно найти в работе [15]:

$$
\square=-4 \sin ^{-2} \theta \partial_{\theta} \sin ^{2} \theta \partial_{\theta}-4 \sin ^{-2} \theta \sin ^{-1} \varphi \partial_{\varphi} \sin \varphi \partial_{\varphi},
$$

где $\theta, \varphi$ - сферические координаты, $\theta \in[0, \pi], \varphi \in(0, \pi)$. Функция $\alpha(\widehat{X})$ задается соотношением

$$
\alpha(\widehat{X})=\alpha_{5}(\theta, \varphi)=\lambda_{1} \cos ^{n} \frac{\theta}{2}+\sum_{i=2}^{3} \lambda_{i}\left(\chi_{i}(\theta, \varphi)\right)^{n / 2}
$$

где

$$
\chi_{i}(\theta, \varphi)=c_{i 1}^{2} \cos ^{2} \frac{\theta}{2}+s_{i 1}^{2} \sin ^{2} \frac{\theta}{2}+c_{i 1} s_{i 1} \sin \theta \cos \varphi
$$


причем $\chi_{i}(\theta, \varphi) \geqslant 0$. Расстояние в паре частиц с номером $i$ в терминах введенных функций выражается как $\left|x_{i}\right|=\rho \sqrt{\chi_{i}(\theta, \varphi)}$.

И для одномерного, и для трехмерного случаев функция $\alpha(\widehat{X})$ удовлетворяет неравенствам

$$
0<\gamma \leqslant \alpha(\widehat{X}) \leqslant \Gamma<\sum_{i} \lambda_{i}
$$

с некоторыми положительными константами $\gamma, \Gamma$.

\section{4. РЕШЕНИЕ УРАВНЕНИЯ ЭЙКОНАЛА ДЛЯ СИСТЕМЫ ТРЕХ ЧАСТИЦ НА ПРЯМОЙ}

Асимптотическое разложение волновой функции $\psi(\rho, \varphi)$ системы трех частиц в $\mathbb{R}^{1}$ на больших расстояниях было построено в работе [11]. Оно имеет вид

$$
\psi(\rho, \varphi) \underset{\rho \rightarrow \infty}{\sim} A(\rho, \varphi) \exp \left\{-\sum_{k=0}^{M} \rho^{1+n / 2-k n} \Phi_{k}(\varphi)\right\},
$$

где $M$ определено соотношением (9). Амплитуда $A(\rho, \varphi)$ и функции $\Phi_{k}(\varphi), k \geqslant 1$, выражены в работе [11] в терминах эйконала $\Phi_{0}(\varphi) \equiv \Phi(\varphi)$. Этот эйконал удовлетворяет нелинейному уравнению

$$
\begin{gathered}
\omega^{2} \Phi^{2}+\left(\Phi^{\prime}\right)^{2}-\alpha_{1}(\varphi)=0, \\
\omega=1+\frac{n}{2}, \quad \Phi(\varphi)=\Phi(\varphi+2 \pi), \quad \Phi(\varphi)>0,
\end{gathered}
$$

которое получается из уравнения (18) в старшем порядке по $\rho$. Однако существование решения $\Phi(\varphi)$ было доказано в работе [11] только для некоторых значений $n$. В этом разделе мы докажем существование решения для произвольного $n$, используя другой подход.

Определим границу уравнением $\gamma(\varphi)=\sqrt{\alpha_{1}(\varphi)} / \omega$. Пусть далее $\Phi(\varphi)$ обозначает некоторое решение уравнения (30), определенное, возможно, только на части интервала $[0,2 \pi]$. Из уравнения (30) следует, что любое такое решение $\Phi(\varphi)$ ограничено, $|\Phi(\varphi)| \leqslant \gamma(\varphi)$.

Исследуем поведение решения $\Phi(\varphi)$ в окрестности точек границы, $\Phi\left(\varphi_{0}\right)=\gamma\left(\varphi_{0}\right)$. Если $\varphi_{0}$ не является особой точкой функции $\gamma(\varphi)$ (т. е. не является минимумом или максимумом), поведение $\gamma(\varphi)$ в окрестности $\varphi_{0}$ имеет вид

$$
\gamma(\varphi) \approx \gamma_{0}+\gamma_{1}\left(\varphi-\varphi_{0}\right) \quad \text { при } \quad \varphi \rightarrow \varphi_{0}
$$

где $\gamma_{0}=\gamma\left(\varphi_{0}\right), \gamma_{1}=\gamma^{\prime}\left(\varphi_{0}\right)$. Будем искать решение $\Phi(\varphi)$ в окрестности такой точки в виде

$$
\Phi(\varphi) \approx \Phi_{0}+a\left|\varphi-\varphi_{0}\right|^{b}
$$

Подставляя представления (31) и (32) в уравнение (30), находим

$$
\Phi_{0}=\gamma_{0}, \quad b=\frac{3}{2}, \quad a^{2}=\frac{8}{9} \omega^{2} \gamma_{0} \gamma_{1} \operatorname{sgn}\left(\varphi-\varphi_{0}\right) .
$$


В окрестности своего локального минимума $\varphi_{0}$ поведение границы $\gamma(\varphi)$ может быть представлено как

$$
\gamma(\varphi) \approx \gamma_{0}+\gamma_{1}\left|\varphi-\varphi_{0}\right|^{l}, \quad \gamma_{0}, \gamma_{1}, l>0
$$

В окрестности таких точек мы по-прежнему будем искать решение $\Phi(\varphi)$ в виде $(32)$. Тогда коэффициенты $\Phi_{0}, a$ и $b$ равны

$$
\begin{array}{ccc}
\Phi_{0}=\gamma_{0}, & \\
b=1+\frac{l}{2}, & a= \pm \frac{\omega}{b} \sqrt{2 \gamma_{0} \gamma_{1}} & \text { для } \quad 0<l<2, \\
b=2, & a=\frac{1}{4}\left(-\omega^{2} \gamma_{0} \pm \omega \sqrt{\omega^{2} \gamma_{0}^{2}+8 \gamma_{0} \gamma_{1}}\right) & \text { для } l=2, \\
b=2, \quad a=-\frac{\gamma_{0} \omega^{2}}{2} \quad \text { или } \quad b=l, a=\gamma_{1} & \text { для } l>2 .
\end{array}
$$

Так как в точке минимума $\gamma_{1}>0$, для любого значения $l$ существует решение $\Phi_{\varphi_{0}}(\varphi)$ с $a>0$, имеющее локальный минимум в точке $\varphi_{0}$.

Определим теперь функцию $\widetilde{\Phi}(\varphi)$ как

$$
\widetilde{\Phi}(\varphi)=\min \left\{\Phi_{\tau}(\varphi) \mid \tau-\text { локальный минимум функции } \gamma(\varphi)\right\} \text {. }
$$

Функция $\widetilde{\Phi}(\varphi)$ является глобальным решением уравнения (30), т. е. она определена и непрерывна на всем интервале $[0,2 \pi]$, хотя ее производная и может иметь скачки на этом интервале.

Докажем сначала, что $\widetilde{\Phi}(t)$ определена для произвольного значения $t \in[0,2 \pi]$. Действительно, рассмотрим два ближайших к неособой точке $t$ минимума $\varphi_{-}$и $\varphi_{+}$ границы $\gamma(\varphi), t \in\left[\varphi_{-}, \varphi_{+}\right]$. Хотя бы на одном из интервалов $\left[\varphi_{-}, t\right]$ или $\left[t, \varphi_{+}\right]$ граница $\gamma(\varphi)$ монотонна, обозначим минимум на этом интервале как $\varphi_{0}$. Рассмотрим функцию $\Phi_{\varphi_{0}}(\varphi)$. Область ее определения может заканчиваться только в тех точках, где выполнено равенство $\Phi_{\varphi_{0}}(\varphi)=\gamma(\varphi)$. Пусть функция $\Phi_{\varphi_{0}}(\varphi)$ не определена для $\varphi=t$. Тогда благодаря ее непрерывности и отсутствию минимумов функции $\gamma(\varphi)$ между точками $\varphi_{0}$ и $t$ область определения функции $\Phi_{\varphi_{0}}(\varphi)$ заканчивается в некоторой неособой точке границы $\hat{\varphi}, \Phi_{\varphi_{0}}(\hat{\varphi})=\gamma(\hat{\varphi})$, которая ближе к $\varphi_{0}$, чем $t$. Таким образом, на интервале $\left[\hat{\varphi}, \varphi_{0}\right]$ функция $\Phi_{\varphi_{0}}(\varphi)$ определена. Однако представление (33) показывает, что в окрестности неособой точки $\hat{\varphi}$ границы решение существует только в направлении удаления от точки $\varphi_{0}$ ближайшего локального минимума границы, так как только тогда $a^{2}>0$. Полученное противоречие показывает, что функция $\widetilde{\Phi}(t)$ определена для всех $t$.

Докажем теперь, что $\widetilde{\Phi}(\varphi)$ - непрерывная функция. Предположим, что $\widetilde{\Phi}$ имеет точку разрыва, и пусть для определенности $\widetilde{\Phi}\left(\varphi_{0}-0\right)<\widetilde{\Phi}\left(\varphi_{0}+0\right) \leqslant \gamma\left(\varphi_{0}\right)$. Тогда решение может быть продолжено из точки $\left(\varphi_{0}-0\right)$ направо как решение $\Phi_{\mathrm{c}}(\varphi)$ уравнения $(30), \Phi_{\mathrm{c}}\left(\varphi_{0}\right)=\widetilde{\Phi}\left(\varphi_{0}-0\right)$. В силу его непрерывности для достаточно малых $\epsilon$ будет выполнено неравенство $\Phi_{\mathrm{c}}\left(\varphi_{0}+\epsilon\right)<\widetilde{\Phi}\left(\varphi_{0}+\epsilon\right)$. Это неравенство противоречит определению (36). Следовательно, наше предположение неверно, и функция $\widetilde{\Phi}(\varphi)$ непрерывна. 
Таким образом, решение $\widetilde{\Phi}(\varphi)$, определенное формулой $(36)$, существует и является непрерывным. Соотношение (36) однозначно задает решение уравнения. Из определения $(25)$ для $\alpha_{1}(\varphi)$ следует, что число минимумов границы $\gamma(\varphi)$ конечно. Следовательно, функция $\widetilde{\Phi}(\varphi)$ построена из конечного числа функций $\Phi_{\varphi_{0}}(\varphi)$. Так как каждая такая функция удовлетворяет уравнению (30), этому уравнению удовлетворяет и функция $\widetilde{\Phi}(\varphi)$.

\section{5. АСИМПТОТИКА ВОЛНОВОЙ ФУНКЦИИ ТРЕХЧАСТИЧНОЙ СИСТЕМЫ В $\mathbb{R}^{3}$}

В этом разделе построено асимптотическое разложение на больших расстояниях волновой функции трехчастичной системы в $\mathbb{R}^{3}$. Вычисления будут проделаны в соответствии с планом, обрисованным в разделе 3 .

Воспользовавшись результатами работы [15], запишем уравнение эйконала (18) в гиперсферических координатах:

$$
\left(S_{\rho}^{\prime}\right)^{2}+\frac{4}{\rho^{2}}\left(\left(S_{\theta}^{\prime}\right)^{2}+\sin ^{-2} \theta\left(S_{\varphi}^{\prime}\right)^{2}\right)-V(X)+E=0
$$

Будем искать его решение $S(\rho, \varphi, \theta)$ при $\rho \rightarrow \infty$ в виде

$$
S(\rho, \varphi, \theta) \approx \sum_{k=0}^{M} S_{k}(\rho, \varphi, \theta), \quad S_{k}=\rho^{\omega_{k}} \Phi_{k}(\varphi, \theta)
$$

Подставляя это разложение в уравнение (37), в старшем порядке по $\rho$ найдем значение $\omega_{0}$ и уравнение для эйконала $\Phi_{0}(\varphi, \theta)$ :

$$
\omega_{0}=1+\frac{n}{2}, \quad \omega_{0}^{2} \Phi_{0}^{2}+4\left(\left(\frac{\partial \Phi_{0}}{\partial \theta}\right)^{2}+\sin ^{-2} \theta\left(\frac{\partial \Phi_{0}}{\partial \varphi}\right)^{2}\right)-\alpha_{5}(\theta, \varphi)=0
$$

Следующие по порядку слагаемые в разложении по $\rho$ определяются требованием обращения в ноль членов в уравнении (37), возникающих из-за наличия энергии $E$. Это требование приводит к значениям $\omega_{k}=\omega_{0}-k n$ и уравнениям для функций $\Phi_{k}(\varphi, \theta)$ :

$$
\omega_{0} \omega_{k} \Phi_{0} \Phi_{k}+4\left(\frac{\partial \Phi_{0}}{\partial \theta} \frac{\partial \Phi_{k}}{\partial \theta}+\sin ^{-2} \theta \frac{\partial \Phi_{0}}{\partial \varphi} \frac{\partial \Phi_{k}}{\partial \varphi}\right)+F_{k}(\varphi, \theta)=0 .
$$

Функции $F_{k}(\varphi, \theta)$ выражаются через предыдущие слагаемые $\Phi_{i}(\varphi, \theta)$ с $i=0, \ldots, k-1$ и при решении уравнения для $\Phi_{k}(\varphi, \theta)$ являются известными функциями. В частности, $F_{1}(\varphi, \theta)=E / 2$. Верхний предел $M$ в сумме (38) выбирается таким образом, чтобы отброшенные слагаемые $S_{k}$ при $k>M$ убывали при $\rho \rightarrow \infty$. Такое значение $M$ вновь определяется соотношением (9).

Амплитуду $A(X)$ можно найти из уравнения $(21)$, которое в обсуждаемом случае может быть записано как

$$
A(\rho, \varphi, \theta)=\mathrm{const} \cdot \rho^{-n / 4}\left(\alpha_{5}(\theta, \varphi)\right)^{-1 / 4}\left(1+O\left(\rho^{-n}\right)\right)
$$


Теперь необходимо доказать существование решения уравнения эйконала (39). Схема доказательства подобна рассуждениям из раздела 4.

Определим границу уравнением $\gamma(\varphi, \theta)=\sqrt{\alpha_{5}(\theta, \varphi)} / \omega_{0}$ и введем обозначение $s(\varphi, \theta) \equiv \Phi_{0}(\varphi, \theta)$. В новых обозначениях уравнение эйконала (39) примет вид

$$
\omega_{0}^{2} s^{2}+4\left(s_{\theta}^{\prime 2}+\sin ^{-2} \theta s_{\varphi}^{\prime 2}\right)=\omega_{0}^{2} \gamma^{2}, \quad s>0
$$

Будем искать решение $s(\varphi, \theta)$ в окрестности границы в виде

$$
s(\varphi, \theta) \approx s_{0}+a_{1}\left|\varphi-\varphi_{0}\right|^{b_{1}}+a_{2}\left|\theta-\theta_{0}\right|^{b_{2}} \quad \text { при } \quad \varphi \rightarrow \varphi_{0}, \quad \theta \rightarrow \theta_{0} .
$$

Необходимо различать два случая:

1) точка границы $\gamma\left(\varphi_{0}, \theta_{0}\right)$ неособая,

$2)$ точка $\gamma\left(\varphi_{0}, \theta_{0}\right)$ является точкой локального минимума границы.

В первом случае в окрестности точки $\left(\varphi_{0}, \theta_{0}\right)$

$$
\gamma(\varphi, \theta) \approx \gamma_{0}+\gamma_{1}\left(\varphi-\varphi_{0}\right)+\gamma_{2}\left(\theta-\theta_{0}\right)
$$

а коэффициенты $s_{0}$ и $a_{i}, b_{i}, i=1,2$, задаются соотношениями

$$
\begin{gathered}
s_{0}=\gamma_{0}, \quad b_{i}=\frac{3}{2}, \\
a_{1}^{2}=\frac{2}{9} \omega_{0}^{2} \gamma_{0} \gamma_{1} \sin ^{2} \theta_{0} \operatorname{sgn}\left(\varphi-\varphi_{0}\right), \quad a_{2}^{2}=\frac{2}{9} \omega_{0}^{2} \gamma_{0} \gamma_{2} \operatorname{sgn}\left(\theta-\theta_{0}\right) .
\end{gathered}
$$

Во втором случае уравнение границы в окрестности точки $\left(\varphi_{0}, \theta_{0}\right)$ может быть записано как

$$
\gamma(\varphi, \theta) \approx \gamma_{0}+\gamma_{1}\left|\varphi-\varphi_{0}\right|^{l_{1}}+\gamma_{2}\left|\theta-\theta_{0}\right|^{l_{2}}, \quad l_{1,2}>0, \quad \gamma_{1,2}>0
$$

Соответствующие коэффициенты в представлении (43) имеют следующие значения:

$$
\begin{array}{rlrl}
s_{0}=\gamma_{0}, & \\
b_{i}=1+\frac{l_{i}}{2}, & a_{1} & = \pm \frac{\omega_{0} \sin \theta_{0}}{b_{1}} \sqrt{\frac{\gamma_{0} \gamma_{1}}{2}}, \\
a_{2} & = \pm \frac{\omega_{0}}{b_{2}} \sqrt{\frac{\gamma_{0} \gamma_{2}}{2}} \\
b_{i}=2, & a_{1}=\frac{\sin ^{2} \theta_{0}}{16}\left(-\omega_{0}^{2} \gamma_{0} \pm \omega_{0} \sqrt{\omega_{0}^{2} \gamma_{0}^{2}+\frac{32 \gamma_{0} \gamma_{1}}{\sin ^{2} \theta_{0}}}\right),
\end{array} \quad \text { для } l_{i}<2,
$$


Если точка является локальным минимумом только по одной из переменных, в ее окрестности нужно использовать оба представления (45), (47) для соответствующих направлений.

Так как для решения всегда можно выбрать константы $a_{1}, a_{2}$ неотрицательными и не равными одновременно нулю, в окрестности каждого локального минимума границы $\gamma$ существует решение уравнения (42), имеющее минимум в этой точке. Обозначим такое решение как $s_{\varphi_{0} \theta_{0}}(\varphi, \theta)$. Определим функцию

$$
\tilde{s}(\varphi, \theta)=\min \left\{s_{\varphi_{0} \theta_{0}}(\varphi, \theta) \mid\left(\varphi_{0}, \theta_{0}\right) \text { - локальный минимум функции } \gamma(\varphi, \theta)\right\} \text {. }
$$

Эта функция определена для всех значений $(\varphi, \theta)$, непрерывна, единственна и удовлетворяет уравнению (42).

Действительно, единственность решения $\tilde{s}(\varphi, \theta)$ немедленно следует из определения (48). Количество минимумов функции $\alpha_{5}(\theta, \varphi)$, заданной в $(27)$, конечно (см. ниже), так что минимум в определении (48) берется по конечному числу функций $s_{\varphi_{0} \theta_{0}}$. Каждая из этих функций удовлетворяет уравнению (42), следовательно, ему же удовлетворяет и функция $\tilde{s}(\varphi, \theta)$.

Непрерывность функции $\tilde{s}(\varphi, \theta)$ доказывается аналогично рассуждениям из раздела 4. Так как каждая функция $s_{\varphi_{0} \theta_{0}}$ непрерывна, разрывы функции $\tilde{s}(\varphi, \theta)$ могут происходить только при переходе от одной функции к другой при взятии минимума в (48). Пусть такой разрыв происходит в точке $(\hat{\varphi}, \hat{\theta})$ при переходе от $s_{(1)}$ к $s_{(2)}$ и $s_{(1)}(\hat{\varphi}, \hat{\theta})<s_{(2)}(\hat{\varphi}, \hat{\theta})$. Продолжая по непрерывности в соответствии с уравнением (42) меньшую функцию $s_{(1)}$ в малую окрестность точки $(\hat{\varphi}, \hat{\theta})$, получим, в силу непрерывности обеих функций и конечности скачка, что в этой окрестности попрежнему $s_{(1)}<s_{(2)}$. Таким образом, мы получаем противоречие с предположением о том, что $(\hat{\varphi}, \hat{\theta})$ - точка перехода от $s_{(1)} \mathrm{K} s_{(2)}$.

Нам еще осталось доказать, что функция $\tilde{s}(\varphi, \theta)$ определена для всех $\varphi, \theta$. Для этого проанализируем сначала расположение экстремумов функции $\alpha_{5}(\theta, \varphi)$. Вычислим ее частную производную по $\varphi$ :

$$
\frac{\partial \alpha_{5}(\theta, \varphi)}{\partial \varphi}=-\frac{n}{2} \sin \theta \sin \varphi \sum_{i=2}^{3} \lambda_{i} c_{i 1} s_{i 1}\left(\chi_{i}(\theta, \varphi)\right)^{n / 2-1}
$$

Экстремумы функции $\alpha_{5}(\theta, \varphi)$ могут находиться в корнях и точках особенностей производной. Особенности производной, если таковые имеются, расположены в точках, где $\chi_{i}(\theta, \varphi)=0$. Эти значения соответствуют $\cos \varphi= \pm 1$, и с учетом области определения угловых координат мы имеем $\varphi=0, \pi$. Значение $\theta$ в точках особенностей определяется соотношением $\operatorname{tg}(\theta / 2)=\left|c_{i 1} / s_{i 1}\right|$, причем в соответствии со знаками коэффициентов $c_{i 1}, s_{i 1}$ [15] каждому значению $\varphi$ отвечает в точности одно значение $\theta$. Экстремумы в точках особенностей, если таковые имеются, обязательно являются минимумами, поскольку $\lambda_{i}>0$. Если особенности отсутствуют, те же точки $\varphi=0, \pi$ являются нулями производной, и в них могут находиться экстремумы функции $\alpha_{5}(\theta, \varphi)$ - как минимумы, так и максимумы. Еще два нуля производной (49) отвечают значениям $\theta=0, \pi$. Значения функции $\alpha_{5}(\theta, \varphi)$ в этих точках не зависят от $\varphi$. 
Помимо указанных выше точек на границе области определения угловых переменных, производная также может обращаться в ноль внутри области, когда сумма в правой части равенства (49) равна нулю. Это условие можно записать в виде

$$
\chi_{2}(\theta, \varphi)=\chi_{3}(\theta, \varphi)\left(-\frac{\lambda_{3}}{\lambda_{2}} \frac{c_{31} s_{31}}{c_{21} s_{21}}\right)^{2 /(n-2)} .
$$

Из данного уравнения можно явно определить $\cos \varphi$ как рациональную функцию от $\cos (\theta / 2)$ и $\sin (\theta / 2)$. Таким образом, для каждого $\theta$ производная обращается в ноль не более чем для одного значения $\varphi$.

Частная производная функции $\alpha_{5}(\theta, \varphi)$ по $\theta$ имеет вид

$$
\begin{aligned}
\frac{\partial \alpha_{5}(\theta, \varphi)}{\partial \theta}= & -\frac{n}{2} \lambda_{1} \cos ^{n-1} \frac{\theta}{2} \sin \frac{\theta}{2}+ \\
& +\sum_{i=2}^{3} \frac{n \lambda_{i}}{2}\left(\chi_{i}(\theta, \varphi)\right)^{n / 2-1}\left(\frac{1}{2}\left(s_{i 1}^{2}-c_{i 1}^{2}\right) \sin \theta+c_{i 1} s_{i 1} \cos \theta \cos \varphi\right) .
\end{aligned}
$$

Экстремумы функции $\alpha_{5}(\theta, \varphi)$ вновь могут находиться на границах области определения угловых переменных. Для того чтобы определить количество нулей функции $(51)$ по переменной $\varphi \in(0, \pi)$ при фиксированном $\theta$, вычислим производную $(51)$ по $\varphi$. Воспользовавшись соотношением (50), найдем, что эта производная пропорциональна $\left(\chi_{3}(\theta, \varphi)\right)^{n / 2-2} \sin \varphi(\cos \varphi+$ const $)$. Первые два множителя в этом выражении не имеют корней внутри интервала $(0, \pi)$, а последний имеет не более одного корня. Таким образом, функция (51) имеет по $\varphi$ не более одного экстремума, следовательно, не может иметь внутри области более двух корней для каждого $\theta$. В силу непрерывности функции только один из этих корней может быть минимумом. Требуя обращения в ноль обеих производных, получаем, что внутри области определения угловых координат имеется не более одного минимума и максимума функции $\alpha_{5}(\theta, \varphi)$ в дополнение к возможным экстремумам на границе области.

Проведенный анализ поведения функции $\alpha_{5}(\theta, \varphi)$ показывает, что произвольная точка $\left(\varphi_{0}, \theta_{0}\right)$ может быть соединена с одной из точек локального минимума $(\hat{\varphi}, \hat{\theta})$ отрезком $i(t)=(\varphi(t), \theta(t)), i(0)=(\hat{\varphi}, \hat{\theta})$ так, что $\gamma(i(t))$ - неубывающая функция. Аналогично рассуждениям из раздела 4 функция $s_{\hat{\varphi} \hat{\theta}}(\varphi, \theta)$ не может пересечься с границей $\gamma(\varphi, \theta)$ на отрезке $i(t)$, так как это противоречило бы представлению (45) и непрерывности границы. Следовательно, функция $s_{\hat{\varphi} \hat{\theta}}(\varphi, \theta)$ определена вплоть до точки $\left(\varphi_{0}, \theta_{0}\right)$.

\section{6. ЗАКЛЮЧЕНИЕ}

В представленной работе построены асимптотические разложения волновой функции на больших расстояниях между частицами для систем двух или трех частиц на прямой $\mathbb{R}^{1}$ и в пространстве $\mathbb{R}^{3}$. Особенностью всех построенных асимптотик является то, что они в существенном определяются лишь потенциалом взаимодействия между частицами.

Действительно, рассмотрим асимптотику функции $\ln |\psi|$. Для всех рассмотренных случаев она в старшем порядке по гиперрадиусу $\rho$ имеет вид

$$
\ln |\psi| \sim-\rho^{1+n / 2} \Phi_{0}(\widehat{X}) \quad \text { при } \quad \rho \rightarrow \infty,
$$


где функция $\Phi_{0}(\hat{x})$ зависит только от углов $\widehat{X}$. Таким образом, в старшем порядке асимптотика не зависит от энергии и определяется лишь степенью конфайнмента $n$. Зависимость от энергии возникает лишь в следующих порядках по гиперрадиусу. Такое поведение $\ln |\psi|$ в этом случае существенно отличается от поведения в случае убывающих парных потенциалов, для которых $\ln |\psi| \sim-\sqrt{-E} \rho$ [1]. Таким образом, для убывающих потенциалов поведение $\ln |\psi|$ определяется энергией системы и не зависит от поведения потенциала при $\rho \rightarrow \infty$.

Предположение о характере поведения парного потенциала (2) на больших расстояниях не является существенным. Если в потенциале присутствуют дополнительные возрастающие слагаемые, они дают дополнительные слагаемые в разложение (38) и модифицируют функции в правой части цепочки уравнений (40). Наиболее быстро растущее слагаемое не изменяется, так что сохраняется и асимптотическое поведение (52).

Нужно заметить, что метод построения асимптотик, описанный в разделах 3 и 5 , можно сравнительно просто обобщить на системы с большим числом частиц. Асимптотические разложения, полученные в настоящей работе, могут быть использованы не только при исследовании моделей барионов [4]-[6], но и, например, для вычисления асимптотик потенциалов кластерных взаимодействий в рамках флип-флоп модели [13].

\section{Список литературы}

[1] С. П. Меркурьев, Л. Д. Фаддеев, Квантовая теория рассеяния для систем нескольких частии, Наука, М., 1985.

[2] С. П. Меркурьев, ТМФ, 32:2 (1977), 187-207.

[3] А.А. Квицинский, Ю.А. Куперин, С. П. Меркурьев, А. К. Мотовилов, С. Л. Яковлев, ЭЧАЯ, 17:2 (1986), 267-317.

[4] Yu. A. Kuperin, A. A. Kvitsinsky, S.P. Merkuriev, E. A. Yarevsky, Nucl. Phys. A, 523:3 (1991), 614-628.

[5] С. М. Герасюта, Ю.А. Куперин, А.В. Саранцев, Е.А. Яревский, ЯФ, 53:5 (1991), $1397-1401$.

[6] B. Silvestre-Brac, Few-Body Syst., 20:1 (1996), 1-25; B. Silvestre-Brac, C. Gignoux, Phys. Rev. D, 32:3 (1985), 743-754.

[7] W. Paul, Rev. Modern Phys., 62:3 (1990), 531-540.

[8] W. Li, L. E. Reichl, Phys. Rev. B, 62:12 (2000), 8269-8275.

[9] Y. Chargui, A. Dhahbi, L. Chetouani, A. Trabelsi, Few-Body Syst., 55:12 (2014), 1233-1243.

[10] J. L. Friar, B. F. Gibson, G. L. Payne, Phys. Rev. C, 22:1 (1980), 284-286.

[11] Yu. A. Kuperin, S. P. Merkuriev, E. A. Yarevsky, J. Math. Phys., 32:1 (1991), 153-156.

[12] В. М. Бабич, В. С. Булдырев, Асимптотические методы в задачах дифракиии коротких волн, Наука, М., 1972.

[13] F. Lenz, J. T. Londergan, E. J. Moniz, R. Rosenfelder, M. Stingl, K. Yazaki, Ann. Phys., 170:1 (1986), 65-254.

[14] М. Абрамовиц, И. Стиган (ред.), Справочник по специальным функциям с формулами, графиками и математическими таблицами, Наука, М., 1979.

[15] A. A. Kvitsinsky, V. V. Kostrykin, J. Math. Phys., 32:10 (1991), 2802-2812. 